\title{
A Reliability Evaluation of Supply Chain: Indicator System and Fuzzy Comprehensive Evaluation
}

\author{
Yuanhong Liu, Haiyan Wu and Ming Luo \\ School of Economics and Management, Nanchang Hangkong University, \\ Nanchang 330063, Jiangxi , P.R. China \\ Lyh682020@163.com birdiwhy@yahoo.com.cn luoming001@sohu.com
}

\begin{abstract}
Reliability evaluation is the basis of the supply chain. Although after 9.11 reliability of supply chain turns to be the focus of the enterprises and the government, little research has been conducted through out the globe. In nature, the supply chain is the integration of material, capital, and information flows. Therefore the evaluation of the supply chain reliability should be considered based on the evaluation of three flows. In view of these ideas, the evaluation of the supply chain reliability is put forwarded in my study, which is achieved by integrating the evaluation of the reliability of the material, capital and information flow with fuzzy comprehensive evaluating theory.
\end{abstract}

Keywords: Supply chain, Reliability, Fuzzy comprehensive evaluation

\section{INTRODUCTION}

Reliability evaluation is the basis of the supply chain. Although after 9.11 reliability of supply chain turns to be the focus of the enterprises and the government, little research has been conducted through out the globe [1-3]. Reliability of supply chain is represented by three specific indicators, which are the delivery performance, the over-ordering and the order fulfillment, according to the SCOR model of the SCC, whereas these three indicators are not integrated to gain the evaluation of supply chain [4]. Other suggestion has been proposed by Jiazhen Huo etc [5]. They preferred to use three quantified indicators, the failed distribution, the delivery punctuality and the customer's complaint to evaluate the supply chain reliability. But they never had considered to integrate these indicators. It is clear that three indicators proposed by the SCC only measures the reliability of material flow. In the further study of Jiazhen Huo, the failed distribution and the delivery punctuality measure the reliability of material flow, the customer complaint measures the reliability of information flow, but it still has some defects. In nature, the supply chain is the integration of material, capital, and information flows [6]. Therefore the evaluation of the supply chain reliability should be considered based on the evaluation of three flows. In view of these ideas, the evaluation of the supply chain reliability is put forwarded in our study, which is achieved by integrating the evaluation of the reliability of the material, capital and information flow with fuzzy comprehensive evaluating theory.

Please use the following format when citing this chapter:

Liu, Y., Wu, H., Luo, M., 2007, in IFIP International Federation for Information Processing, Volume 255, Research and Practical Issues of Enterprise Information Systems II Volume 2, eds. L. Xu, Tjoa A., Chaudhry S. (Boston: Springer), pp. 1515-1521. 


\section{RELIABILITY EVALUATION OF THE SUPPLY CHAIN}

It is the material, information and capital flows that connect the customers and the supply chain. We discuss how to establish the evaluation indicator system of the supply chain reliability, from the material flow, information flow and capital flow aspects.

\subsection{Measure the Material Flow Reliability}

(1) Eligible delivery: it reflects the level of delivery quality during a certain period, which can be quantified with a ratio: the number of Eligible goods delivered / the number of all goods delivered

(2) On time delivery: it reflects whether the goods are delivered on time during a certain period, which can be quantified with a ratio: The number of times that goods are delivery / the times of total delivery

(3) Order fulfillment: it refers to a fraction of the number of products produced and the number of the products in demand.

\subsection{Measure the Capital Flow Reliability}

The capital reliability of the supply chain reflects the reliability of the capital flow between the supply chain and customers, which can be measured by three specific indicators, namely doubtful accounts, account sale tick, exchange rate risks.

(1)Doubtful accounts: doubtfiul account reflects the rate of collection of accounts receivable. The verification and computation of doubtful accounts is firmly carried out according to the financial and accounting system.

(2)Account sale tick: an account sale tick reflects the timely gathering during a certain period, which can be defined by the ratio of an account sale tick in total sales

(3)Exchange rate risks: exchange rate risk reflects the risk of transnational gathering, which is a qualitative indicator.

(4) Order fulfillment: it refers to a fraction of the number of products produced and the number of the products in demand.

\subsection{Measure Information Flow Reliability}

Reliability of information flow in the supply chain reflects the information flow between the customers and the supply chain. Be aware of the bidirectional flow of the information which can be measured by two indicators

(1) The timeliness of the information delivered.

It reflects the timely communication between customers and the supply chain, which can be computed with a ratio: the number of times that information is delivered on time / in total number of times that information is delivered.

(2) Precision of the information delivered. 
It reflects precision of the information delivered, which can be represented by the number of times that precise information is delivered in total number of times that information is delivered.

From the above, we can gain a multilevel tree of the reliability evaluation indicator system for the supply chain (see figure 1).

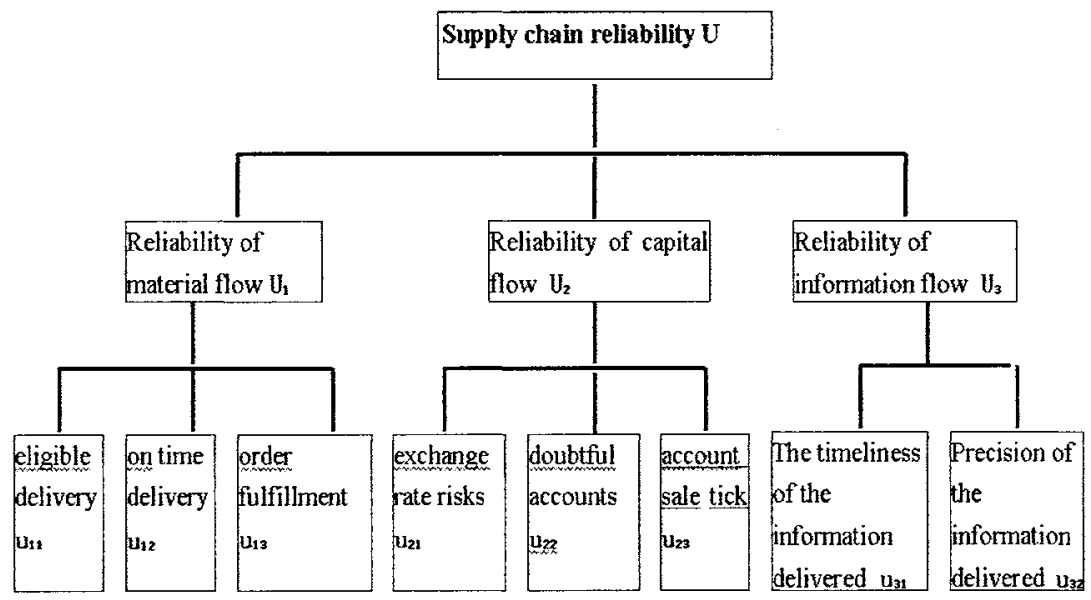

Figure 1. Reliability Indicator System of the Supply Chain

\section{THE FUZZY COMPREHENSIVE EVALUATION OF THE SUPPLY CHAIN RELIABILITY}

Fuzzy comprehensive evaluation is based on the fuzzy set theory. Fuzzy set theory deals with the representation of classes whose boundaries are not well defined. The key idea is to associate a membership function with the elements of the class. The evaluation of supply chain reliability involves lots of uncertainty. The adoption of the fuzzy set theory should objectively and reasonably represent the performance of supply chain.

\subsection{The Definition of the Indicator Sets and Subsets}

From the figure 1, we get: 
Three first level indicators, which is represented as set $U=\{U 1, U 2, U 3\}$, where subset $U 1$ is the reliability of the material flow, subset $U 2$ is the reliability of the capital flow, subset $\mathrm{U} 3$ is the reliability of the information flow.

Eight second level indicators are proposed right after the first level indicators are set, which are the components of three subsets $\mathrm{U} 1, \mathrm{U} 2$, and $\mathrm{U} 3$.

And let $\mathrm{U} 1$ be $\mathrm{U} 1=\{\mathrm{u} 11, \mathrm{u} 12, \mathrm{u} 13\}$, where $\mathrm{u} 11$ is the eligible products delivered, $\mathrm{u} 12$ is the timeliness of the delivery, $\mathrm{u} 13$ is the order fulfillment.

Let $\mathrm{U} 2$ be $\mathrm{U} 2=\{\mathrm{u} 21, \mathrm{u} 22, \mathrm{u} 23\}$, where $\mathrm{u} 21$ is the doubtful accounts, $\mathrm{u} 22$ is exchange rate risks, $\mathrm{u} 23$ is account sale tick.

Let $\mathrm{U} 3$ be $\mathrm{U} 3=\{\mathrm{u} 31, \mathrm{u} 32\}$, where $\mathrm{u} 31$ is the on time delivery of information, $\mathrm{u} 32$ is the precise information delivered.

\subsection{Compute the Weights of the Indicators Using AHP or Delphi}

The weight matrix $A=(A 1, A 2, A 3)$ is composed of three first level indicators.

The secondary matrices $\mathrm{W} 1=(\mathrm{w} 11$, w12 ,w13), $\mathrm{W} 2=(\mathrm{w} 21$,w22 ,w23), $\mathrm{W} 3=(\mathrm{w} 31, \mathrm{w} 32)$ are composed of eight second level indicators.

\subsection{Compute Grading Matrix V of the Supply Chain}

Grading matrix V should reflect the rank of reliability of the supply chain and provide information to the decision maker. The reliability are categorized into three levels (high, medium, low), which comprises the grading matrix $\mathrm{V}=\{\mathrm{H}, \mathrm{M}, \mathrm{L}\}$.

\subsection{Model the Membership Function of the Second Level Indicators Using Econometrics, and then Quantify the Row Vector of the Membership}

Grading matrix V should reflect the rank of reliability of the supply chain and Let's illustrate how to measure the second level indicator, say, doubtful accounts $\mathbf{u} 21$.

Suppose the following membership function of doubtful accounts can be defined as: High membership function:

$$
d_{211}\left(u_{21}\right)=\left\{\begin{array}{rc}
1, & u_{21} \leq 1 \% \\
\frac{3 \%-u_{21}}{3 \%-1 \%}, & 1 \%<u_{21}<3 \% \\
0, & u_{21} \geq 3 \%
\end{array}\right.
$$

Medium membership function: 
A Reliability Evaluation of Supply Chain: Indicator System and Fuzzy Comprehensive Evaluation 1519

$$
d_{212}\left(u_{21}\right)=\left\{\begin{array}{rc}
0, & u_{21}<1 \% \\
\frac{u_{21}-1 \%}{3 \%-1 \%}, & 1 \% \leq u_{21}<3 \% \\
1, & 3 \% \leq u_{21}<5 \% \\
\frac{7 \%-u_{21}}{7 \%-5 \%}, & 5 \% \leq u_{21}<7 \% \\
0, & u_{21} \geq 7 \%
\end{array}\right.
$$

Low membership function:

$$
d_{213}\left(u_{21}\right)=\left\{\begin{aligned}
0, & u_{21} \leq 5 \% \\
\frac{u_{21}-5 \%}{7 \%-5 \%}, & 5 \%<u_{21}<7 \% \\
1, & u_{21} \geq 7 \%
\end{aligned}\right.
$$

The distribution of the membership value is showed in figure 2 .

If doubtful accounts $=6 \%$, the row vector of the membership ( $u 21 / \mathrm{H}, \mathrm{u} 21 / \mathrm{M}, \mathrm{u} 21 / \mathrm{L}$ ) $=(\mathrm{d} 211, \mathrm{~d} 212, \mathrm{~d} 213)=(0.5,0.5,0)$;

If doubtful accounts $=0.5 \%$, the row vector of the membership (d211, d212, $\mathrm{d} 213)=(1,0,0)$;

The computations attached to other indicators in the second level are analogous.

3.5 Row Vectors of the Second Level Indicators Are Combined into Three Membership Matrices

$$
D_{1}=\left(\begin{array}{lll}
d_{111} & d_{112} & d_{113} \\
d_{121} & d_{122} & d_{123} \\
d_{131} & d_{132} & d_{133}
\end{array}\right)
$$




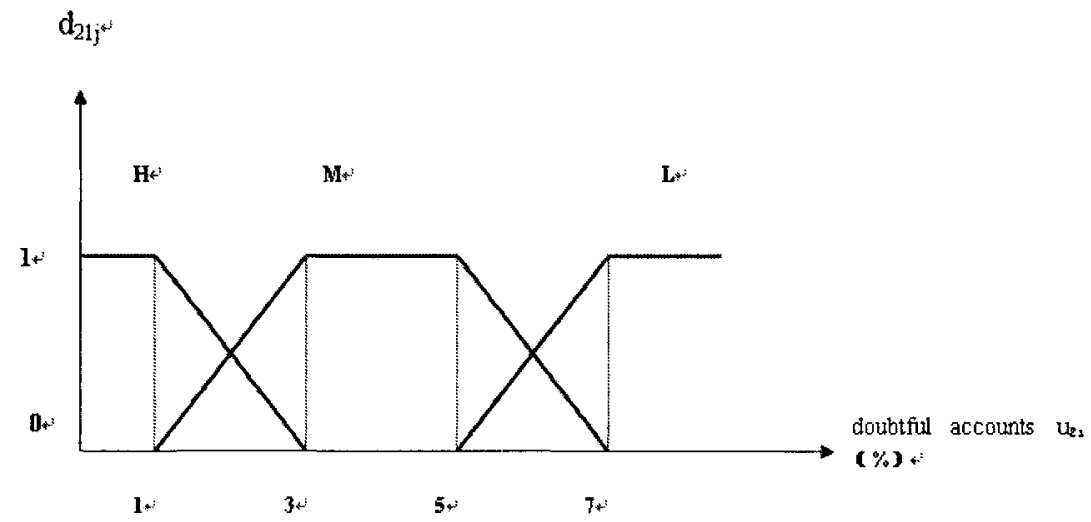

Figure 2. Doubtful Accounts Membership Function

$$
\begin{gathered}
D_{2}=\left(\begin{array}{lll}
d_{211} & d_{212} & d_{213} \\
d_{221} & d_{222} & d_{223} \\
d_{231} & d_{232} & d_{233}
\end{array}\right) \\
D_{3}=\left(\begin{array}{ll}
d_{311} & d_{312} \\
d_{321} & d_{322}
\end{array}\right)
\end{gathered}
$$

\subsection{Compute Matrix $D$ on the Basis of $D_{1}, D_{2}$, and $D_{3}$}

Quantify the second membership vector using appropriate composite operator, which can be gained by computing the membership matrices of the second level indicators and weight matrices, and compute matrix D of the first level indicators.

Reliability of material flow:

$$
R_{1}=W_{1} \circ D_{1}=\left(\begin{array}{lll}
d_{11} & d_{12} & d_{13}
\end{array}\right)
$$

Reliability of capital flow:

$$
R_{2}=W_{2} \circ D_{2}=\left(\begin{array}{lll}
d_{21} & d_{22} & d_{23}
\end{array}\right)
$$

Reliability of information flow:

$$
R_{3}=W_{3} \circ D_{3}=\left(\begin{array}{lll}
d_{31} & d_{32} & d_{33}
\end{array}\right)
$$


A Reliability Evaluation of Supply Chain: Indicator System and Fuzzy

$$
D=\left(\begin{array}{lll}
d_{11} & d_{12} & d_{13} \\
d_{21} & d_{22} & d_{23} \\
d_{31} & d_{32} & d_{33}
\end{array}\right)
$$

\subsection{Compute the Membership Vector $R$ for the Reliability of Supply Chain}

Compute the membership vector $\mathrm{R}$ for the reliability of supply chain, using the membership matrices and the corresponding weight matrices of the first level indicators.

Reliability of the supply chain:

$$
R=A \circ D=\left(\begin{array}{lll}
R_{I} & R_{M} & R_{L}
\end{array}\right)
$$

\section{CONCLUSIONS}

A reliability evaluation indicator system is proposed in the paper, using the fuzzy comprehensive evaluation, from material flow, capital flow and information flow aspects. Though it is practical in some scenario, but it still needs improvement and further research on it.

\section{REFERENCES}

1. D. Luton, Inventory Accuracy: An overlooked component of supply chain reliability, Materials Management and Distribution. Volume 49, Number 7, pp.71, (2004).

2. Anonymous, Supply Chain Reliability, Chain Store Age. Volume 80, Number 7, pp.61, (2004).

3. S.Y. Sohn and I.S. Choi, Fuzzy QFD for supply chain management with reliability consideration, Reliability Engineering and System Safety. Volume 72, Number 2, pp.327334, (2001).

4. Anonymous, Supply-Chain Operations Reference-model, Supply-Chain Council (2005). http://www.supply-chain.org/slides/SCOR Overview 6.1.pdf(Accessed May 18, 2005).

5. J. Huo, M. Sui, and Z. Liu, Construction of Integrated Supply Chain Performance Measurement System, Journal of Tongii University. Volume 30, Number 4, pp.495-499, (2002).

6. S. Ma, Y. Lin, and Z. Chen, Supply Chain Management (China Machine Press: Beijing, 2000). 